\title{
Relapse-associated microRNA in gastric cancer patients after $S-1$ adjuvant chemotherapy
}

\author{
TETSUYA OMURA $^{1}$, YUTAKA SHIMADA ${ }^{1}$, TAKUYA NAGATA ${ }^{1}$, TOMOYUKI OKUMURA $^{1}$, \\ JUNYA FUKUOKA ${ }^{2}$, FUMINORI YAMAGISHI ${ }^{3}$, SADAKATSU TAJIKA ${ }^{4}$, \\ SANAE NAKAJIMA $^{5}$, ATSUSHI KAWABE ${ }^{5}$ and KAZUHIRO TSUKADA ${ }^{1}$
}

\begin{abstract}
Departments of ${ }^{1}$ Surgery and Science, and ${ }^{2}$ Surgical Pathology, University of Toyama, Toyama 930-0194; ${ }^{3}$ Department of Surgery, Itoigawa General Hospital, Itoigawa, Niigata 941-8502; ${ }^{4}$ Department of Surgery, Saiseikai Toyama Hospital, Toyama 931-8533; ${ }^{5}$ Department of Surgery, Murakami Memorial Hospital, Asahi University, Gifu 500-8523, Japan
\end{abstract}

Received October 24, 2013; Accepted November 18, 2013

DOI: 10.3892/or.2013.2900

\begin{abstract}
S-1 has been recommended as adjuvant chemotherapy in patients after curative surgery for gastric cancer. However, some patients suffer recurrence even after S-1 adjuvant chemotherapy. The present study was conducted to find a predictive marker of the efficacy of S-1 adjuvant chemotherapy. We examined the microRNA (miRNA) expression of 35 patients who underwent $\mathrm{S}-1$ adjuvant chemotherapy after curative surgery (R0) for pathological stage II or III gastric cancer. miRNAs were extracted from formalin-fixed, paraffinembedded specimens for analysis and miRNA expression was examined using miRNA oligo chips. Fifteen patients relapsed and 20 did not over 5 years. Five miRNAs (miR-92b, 422a, $4732-5 p, 4758-3 p$ and 221 ) were highly expressed according to the tumor/normal $(\mathrm{T} / \mathrm{N})$ ratio in the patients who relapsed but not in those who did not relapse (P-value $<0.05)$ by microarray analysis. If tumors showed high expression of 4 miRNAs (miR-92b, 422a, 4732-5p and 4758-3p) their positive predictive value of relapse was $93.8 \%$ and negative predictive value was $92.3 \%$. In this case, their disease-free survival rate and overall survival rate were very poor. Our findings indicate that miR-92b, miR-422a, miR-4732-5p and miR-4758-3p are closely associated with relapse following S-1 adjuvant chemotherapy in gastric cancer.
\end{abstract}

\section{Introduction}

Gastric cancer is a common malignancy worldwide, with eastern Asia considered to have the highest mortality rate. The mainstay of treatment for gastric cancer is surgery (1). However, some patients experience a recurrence after curative resection for advanced gastric cancer. Various regimens with

Correspondence to: Dr Tetsuya Omura, Department of Surgery and Science, University of Toyama, 2630 Sugitani, Toyama 930-0194, Japan

E-mail: d0961003@ems.u-toyama.ac.jp

Key words: relapse, microRNA, S-1, gastric cancer 5-fluorouracil (5-FU) have shown that adjuvant chemotherapy is effective against gastric cancer (2-4). 5-FU is the principal component of S-1 (TS-1; Taiho Pharmaceutical Co., Ltd., Tokyo, Japan). S-1 is an orally active combination of tegafur (a prodrug that is converted by cells to fluorouracil), gimeracil (an inhibitor of dihydropyrimidine dehydrogenase, which degrades fluorouracil), and oteracil (which inhibits the phosphorylation of fluorouracil in the gastrointestinal tract, thereby reducing the gastrointestinal toxic effects of fluorouracil). The Adjuvant Chemotherapy Trial of S-1 for Gastric Cancer (ACTS-GC) group reported on the effects of postoperative $\mathrm{S}-1$ chemotherapy compared with surgery alone in pathological stage II or III gastric cancer patients (5). Since then, S-1 chemotherapy has been recommended as adjuvant chemotherapy for patients who have undergone curative surgery for advanced gastric cancer (6).

Nevertheless, some patients suffer recurrence even after S-1 adjuvant chemotherapy $(5,7)$. There was no clear predictive marker of its effects. If we identify predictors of relapse after adjuvant chemotherapy, patients may be able to receive more suitable regimen in their cancer characteristics and avoid both side-effects and excessive therapy.

microRNAs (miRNAs) regulate physiological processes, cell and differentiation, and apoptosis through the suppression of mRNA (8). As miRNAs are associated with these tumor characteristics, specific miRNAs are thought to be associated with sensitivity to chemotherapy $(9,10)$.

We previously extracted high quality miRNAs from formalin-fixed, paraffin-embedded (FFPE) samples obtained from gastric cancer patients who had undergone operations, excluded stage I and IV in non-radical surgery (11) and we detected specific miRNAs associated with the prognosis of these patients using high-sensitivity DNA chips. Therefore, we hypothesized that patients who may require further chemotherapy can be identified by miRNA expression profile analysis in patients who showed relapse after adjuvant chemotherapy for gastric cancer.

\section{Materials and methods}

Patients and tissue specimens. FFPE specimens of gastric cancer and associated patient information were collected. 
Table I. Characteristics of the evaluable gastric cancer patients.

\begin{tabular}{|c|c|c|c|}
\hline Characteristics & Relapse $(n=15)$ & Non-relapse $(n=20)$ & P-value \\
\hline Age (years, mean \pm SD) & $71.0 \pm 2.88$ & $63.4 \pm 2.49$ & 0.0543 \\
\hline \multicolumn{4}{|l|}{ Gender } \\
\hline Male & 13 & 12 & \multirow[t]{2}{*}{0.0746} \\
\hline Female & 2 & 8 & \\
\hline \multicolumn{4}{|l|}{ Histological type } \\
\hline Differentiated $^{\mathrm{a}}$ & 6 & 8 & \multirow[t]{2}{*}{1.0000} \\
\hline Undifferentiated $^{\mathrm{b}}$ & 9 & 12 & \\
\hline Maximum tumor diameter (mm, mean) & 62 & 48 & 0.0639 \\
\hline \multicolumn{4}{|l|}{ Depth of invasion } \\
\hline $\mathrm{T} 2$ & 1 & 6 & \multirow[t]{2}{*}{0.0716} \\
\hline$\geq \mathrm{T} 3$ & 14 & 14 & \\
\hline \multicolumn{4}{|l|}{ Lymph node metastasis } \\
\hline$<3$ & 6 & 10 & \multirow[t]{2}{*}{0.5560} \\
\hline$\geq 3$ & 9 & 10 & \\
\hline \multicolumn{4}{|l|}{ p-stage } \\
\hline II & 4 & 9 & \multirow[t]{2}{*}{0.2623} \\
\hline III & 11 & 11 & \\
\hline \multicolumn{4}{|l|}{ Carcinoembryonic antigen (ng/ml) } \\
\hline$<5$ & 12 & 13 & \multirow[t]{2}{*}{0.2502} \\
\hline$\geq 5$ & 2 & 7 & \\
\hline \multicolumn{4}{|l|}{ Carbohydrate antigen 19-9 (U/ml) } \\
\hline$<37$ & 14 & 17 & \multirow[t]{2}{*}{0.2513} \\
\hline$\geq 37$ & 0 & 3 & \\
\hline S-1 total dose (mg, mean) & 15970 & 22263 & 0.1892 \\
\hline
\end{tabular}

${ }^{\mathrm{a} D i f f e r e n t i a t e d, ~ p a p i l l a r y ~ o r ~ t u b u l a r ~ a d e n o c a r c i n o m a . ~}{ }^{\mathrm{b}}$ Undifferentiated, poorly differentiated or mucinous adenocarcinoma, signet-ring cell carcinoma.

In total, 458 patients with p-stage II or III gastric cancer underwent curative surgery between 2000 and 2012, including 161 patients from Toyama University Hospital, 114 from Itoigawa General Hospital, 126 from Saiseikai Toyama Hospital and 57 from Murakami Memorial Hospital Asahi University; the latter 57 patients underwent surgery between 2006 and 2012. Of these, 398 were ineligible as they did not undergo adjuvant chemotherapy (173 patients), were treated with a chemotherapy other than S-1 (105 patients), underwent S-1 chemotherapy for $<3$ months (22 patients), died from a cause other than gastric cancer (1 case), underwent neoadjuvant chemotherapy (20 cases), were followed up for $<5$ years (52 cases), or relapsed in $<6$ months (10 cases). S-1 treatment was continued for at least 3 months in 452 patients $(87.4 \%)$ in the ACTS-GC study (5). Therefore, we excluded patients treated with $\mathrm{S}-1$ for $<3$ months and $\mathrm{T} 1$ cases were excluded as the tumors were too small for extraction of miRNA (15 patients). Thus, a total of 60 specimens were selected from 458 gastric cancer patients. We obtained approval from the Ethics Committee of the University of Toyama (Approval number, 20-63), Itoigawa General Hospital, Saiseikai Toyama Hospital, and Murakami Memorial Hospital Asahi University.
RNA extraction from FFPE specimens. RNA was extracted from FFPE specimens of tumor and normal tissue from each patient, as previously reported (12). Extracted RNA was processed using a silica-based spin column (Toray Industries, Tokyo, Japan) to obtain purified total RNA. The degree of RNA cross-linking and RNA degradation was analyzed using electrophoresis with an Agilent 2100 Bioanalyzer (Agilent Technologies, Santa Clara, CA, USA). The RNA quality criteria were as previously reported (12). In addition, we analyzed samples with the same degree of total RNA decay in this experiment so as to examine the relationship between tumor and normal tissues. Thus, we determined whether RNAs could be used for microarray analysis.

miRNA assays. RNAs were labeled with Hy5 (miRCURY LNA microRNA Hy5 Power labeling kit; Exiqon A/S Corp., Vedbaek, Denmark). miRNAs were examined with Toray 3D-Gene ${ }^{\circledR}$ miRNA oligo chips (ver. 17; Toray Industries). A solution adjusted for the DNA chips was applied, (including $500 \mathrm{ng}$ of total RNA) and hybridization was performed. Fluorescent signals were scanned using a 3D-Gene Scanner (Toray Industries) and analyzed with 3D-Gene Extraction software 
Table II. miRNA expression of 35 patients analyzed using miRNA oligo chips.

\begin{tabular}{|c|c|c|c|c|c|}
\hline Name & Relapse signal & Relapse $\mathrm{T} / \mathrm{N}$ ratio & Non-relapse signal & Non-relapse $\mathrm{T} / \mathrm{N}$ ratio & P-value \\
\hline hsa-miR-92b & 15 & 1.27 & 20 & 0.95 & 0.0143 \\
\hline hsa-miR-422a & 15 & 0.89 & 20 & 0.61 & 0.0174 \\
\hline hsa-miR-4732-5p & 15 & 2.00 & 20 & 1.09 & 0.0203 \\
\hline hsa-miR-4758-3p & 15 & 1.36 & 20 & 0.98 & 0.0218 \\
\hline hsa-miR-221 & 15 & 1.61 & 20 & 1.03 & 0.0384 \\
\hline hsa-miR-1246 & 15 & 1.98 & 20 & 1.06 & 0.0703 \\
\hline hsa-miR-3663-3p & 15 & 1.03 & 20 & 0.86 & 0.0764 \\
\hline hsa-miR-3135b & 15 & 1.03 & 20 & 0.80 & 0.0845 \\
\hline hsa-miR-30d & 15 & 1.06 & 20 & 0.68 & 0.0993 \\
\hline
\end{tabular}

Table III. Cut-off value for predicting relapse analyzed using receiver operating characteristic (ROC) curves.

\begin{tabular}{|c|c|c|c|c|c|c|c|c|}
\hline Name & Cut-off & AUC & Sensitivity & Specificity & True positive & True negative & False positive & False negative \\
\hline hsa-miR-92b & 1.046232 & 0.75 & 0.93 & 0.6 & 14 & 12 & 8 & 1 \\
\hline hsa-miR-422a & 0.815585 & 0.75 & 0.73 & 0.85 & 11 & 17 & 3 & 4 \\
\hline hsa-miR-4732-5p & 1.146845 & 0.77 & 0.73 & 0.75 & 11 & 15 & 5 & 4 \\
\hline hsa-miR-4758-3p & 1.028134 & 0.75 & 0.80 & 0.65 & 12 & 13 & 7 & 3 \\
\hline hsa-miR-221 & 1.000008 & 0.70 & 0.80 & 0.65 & 12 & 13 & 7 & 3 \\
\hline hsa-miR-1246 & 1.223516 & 0.67 & 0.73 & 0.7 & 11 & 14 & 6 & 4 \\
\hline hsa-miR-3663-3p & 0.778234 & 0.70 & 0.93 & 0.45 & 14 & 9 & 11 & 1 \\
\hline hsa-miR-3135b & 1.170270 & 0.65 & 0.40 & 0.95 & 6 & 19 & 1 & 9 \\
\hline hsa-miR-30d & 1.068176 & 0.63 & 0.53 & 0.85 & 8 & 17 & 3 & 7 \\
\hline
\end{tabular}

(Toray Industries). Quality evaluations were performed for the averages of the tumor/normal ( $\mathrm{T} / \mathrm{N}$ ratio) as previously reported (12).

Statistical analysis. P-values for gender, histological type, depth of invasion, lymph node metastasis, p-stage, preoperative serum carcinoembryonic antigen (CEA) levels and pre-operative serum carbohydrate antigen 19-9 (CA 19-9) levels were calculated using the Chi-square test. P-values for age, maximum tumor diameter and the total S-1 dose were calculated using the Student's t-test. The T/N ratio between the groups that did and did not relapse were calculated and compared with the Student's t-test. We constructed receiver operating characteristic (ROC) curves and calculated the area under the curve (AUC) to evaluate the specificity and sensitivity of predicting cases. We divided gastric cancer patients into two groups: the miRNA high-expression group and the low-expression group, according to ROC curves. Positive predictive value and negative predictive value were calculated as previously reported (13). The disease-free survival curve and overall survival curve were plotted according to the Kaplan-Meier method. The differences in survival rates among the groups with high and low miRNA expression were analyzed using the log-rank test and the generalized Wilcoxon test. Disease-free survival time was calculated from the date of surgery until the patient relapsed or until the last follow-up. Overall survival time was calculated from the date of surgery until the patient succumbed to the disease or until the last follow-up. All statistical analyses were performed using Microsoft Office Excel 2007 and JMP 10. P<0.05 was considered to indicate a statistically significant result.

\section{Results}

RNA was extracted from tumor and normal tissue specimens obtained from 60 patients. Twenty-five cases were excluded due to poor RNA quality. Of the remaining 35 cases, 15 relapsed within 5 years. Characteristics of the patients are shown in Table I. There were no significant differences in age, gender, histological type, maximum tumor diameter, depth of invasion, lymph node metastasis, $\mathrm{p}$-stage, pre-operative serum CEA levels, pre-operative serum CA 19-9 levels, or S-1 total dose between patients who relapsed and those who did not.

A total of 218 miRNAs were evaluated for $\mathrm{T} / \mathrm{N}$ ratio in each of the 15 relapse and 20 non-relapse cases. For these 218 miRNAs, microarray analysis revealed 5 miRNAs (miR-92b, $422 \mathrm{a}, 4732-5 \mathrm{p}, 4758-3 \mathrm{p}$ and 221) that were highly expressed according to the $\mathrm{T} / \mathrm{N}$ ratio in the patients who relapsed but not in those who did not relapse (P-value $<0.05$ ), as shown in Table II (from a group of 9 miRNAs). The results of ROC curves are shown in Table III. We divided 35 gastric cancer patients into two groups, the miRNA high-expression group and the lowexpression group, according to ROC curves. Cluster analysis of 5 miRNAs (miR-92b, 422a, 4732-5p, 4758-3p and 221) clas- 


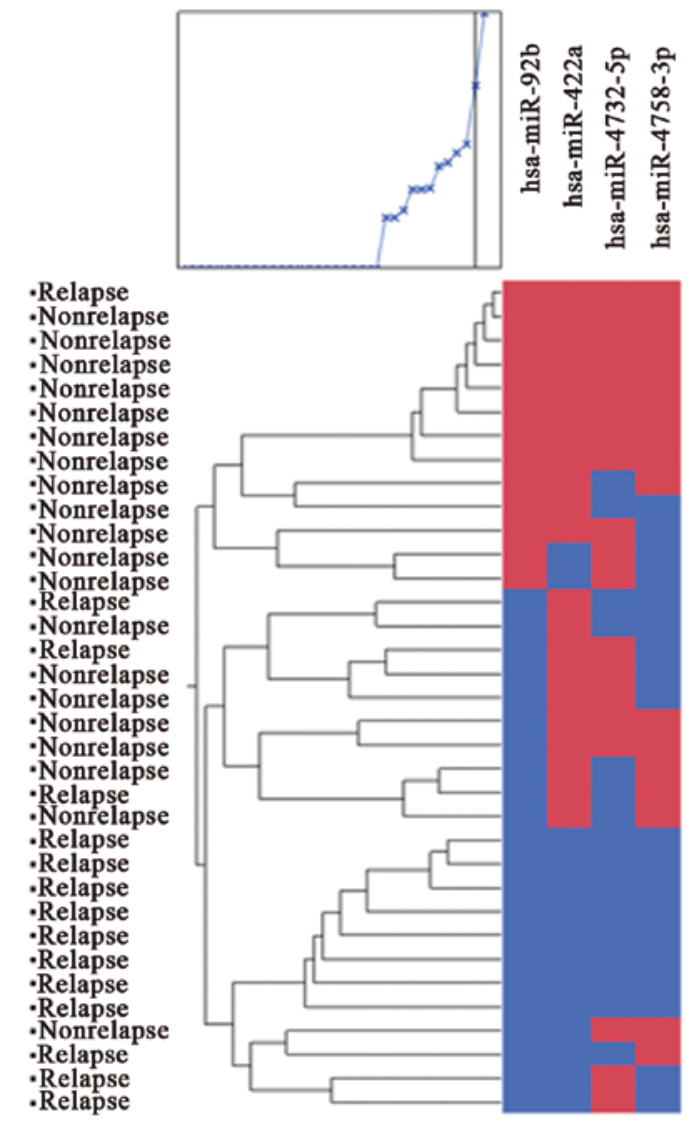

Figure 1. Cluster analysis of 4 miRNAs (miR-92b, 422a, 4732-5p and 4758-3p). We divided 35 gastric cancer patients into two groups, the miRNA high-expression group (blue) and the low-expression group (red), according to ROC curves. Cluster analysis of 4 miRNAs (miR-92b, 422a, 4732-5p and 4758-3p) classified two groups. Positive predictive value was $93.8 \%$. Negative predictive value was $92.3 \%$.

sified two groups. Their positive predictive value was $85.1 \%$ and the negative predictive value was $82.4 \%$. Cluster analysis of 4 miRNAs (miR-92b, 422a, 4732-5p and 4758-3p) classified two groups (Fig. 1). Their positive predictive value was $93.8 \%$ and negative predictive value was $92.3 \%$. If tumors showed high expression of 4 miRNAs (miR-92b, 422a, 4732-5p and 4758-3p), their disease-free survival rate and overall survival rate were very poor (Fig. 2). We searched for potential target of mRNA using bioinformatic logarithms available on-line (TargetScan). According to TargetScan, dihydropyrimidine dehydrogenase gene (DPYD) may be one of the predictable target genes of miR-92b (Fig. 3).

\section{Discussion}

In the present study, we identified 5 specific miRNAs related to gastric cancer recurrence after adjuvant $S-1$ chemotherapy. If tumors showed high expression of 4 miRNAs, miR-92b, 422a, 4732-5p and 4758-3p, their disease-free survival rate and overall survival rate were very poor. Combination of miRNAs may be possible to predict recurrence after S-1 chemotherapy.

No study has reported the relationship between gastric cancer, S-1 resistance, and miRNAs. In the study of the relationship between $\mathrm{S}-1$ resistivity and miRNAs in colon cancer, Nakajima et al (14) reported that let-7g and miR-181b
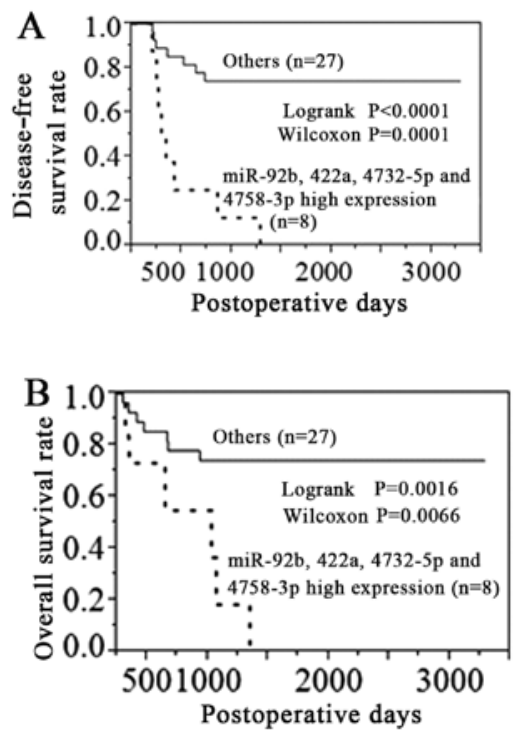

Figure 2. Disease-free survival and overall survival curves regarding 4 miRNAs (miR-92b, 422a, 4732-5p and 4758-3p). (A) Disease-free survival time was calculated from the date of surgery until the patient relapsed or until the last follow-up. (B) Overall survival time was calculated from the date of surgery until the patient succumbed to the disease or until the last follow-up. If tumors showed high expression of 4 miRNAs (miR-92b, 422a, 732-5p and $4758-3 p$ ), their prognosis was very poor. $\mathrm{P}<0.05$ was considered to indicate a statistically significant difference.

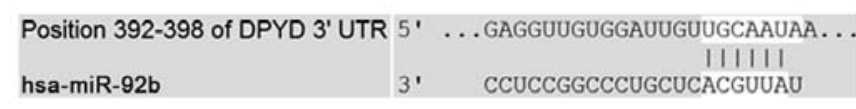

Figure 3. Analysis of miR-92b binding sites in the DPYD gene. Homology between the 3'-UTR of DPYD and the predicted target sequence of miR-92b. miR-92b is one of the potential targets within the 3'-UTR of DPYD mRNA.

are strongly associated with the response to S-1 chemotherapy. In their report, 69.6\% (32/46) patients underwent S-1 chemotherapy and $30.4 \%(14 / 46)$ patients underwent S-1 plus cisplatin chemotherapy. Thus, the results of their study may be affected by cisplatin combination therapy.

There are no previous reports on the relationship between miR-92b expression and gastric cancer recurrence after adjuvant S-1 chemotherapy. In addition, no study has reported the relationship between $\mathrm{S}-1$ and miR-92b expression or between gastric cancer and miR-92b expression. Haug et al (15) reported that MYCN-regulated miRNA-92 inhibits the secretion of the tumor suppressor Dickkopf-3 (DKK3) in neuroblastoma. Also, Wang et al (16) reported that miR-92b binds to 3'UTR of Nemolike kinase that acts as a negative regulator of Wnt signaling and activates $W n t / \beta$-catenin signaling. As a result, miR-92b activates glioma proliferation and invasion. Since miR-92b was highly expressed according to the $\mathrm{T} / \mathrm{N}$ ratio in the patients who relapsed, it may be considered to promote tumor cell growth.

Dihydropyrimidine dehydrogenase gene (DPYD) is key in the antitumor effect of 5-fluorouracil and S-1. Dihydropyrimidine dehydrogenase (DPD) is the rate-limiting enzyme in the catabolism of pyrimidine. However, Ichikawa et al (17) and Jeung et al (18) reported that S-1 responders had higher DPD expression than S-1 non-responders in gastric cancer patients. If miR-92b downregulates this gene, these reports support our 
results that miR-92b was highly expressed according to the $\mathrm{T} / \mathrm{N}$ ratio average in the gastric cancer patients who relapsed but not in those who did not relapse after S-1 chemotherapy. Kai et al (19) reported that 5-FU responders showed lower DPD expression than 5-FU non-responders in gastric cancer patients. Regarding DPD expression, there might be a difference between S-1 therapy and 5-FU therapy.

In the study of the relationship between miR-422a and cancer, Gougelet et al (20) reported that miR-422a was overexpressed in good responders for ifosfamide therapy in osteosarcoma. Faltejskova et al (21) reported that miR-422a was significantly decreased in colorectal cancer (CRC) tissues compared to normal tissues. Thus, these results differed from our observation that miR-422a was overexpressed in the relapse group. These findings together indicate a dual role of miR-422a as either a tumor-promoting or a tumor-suppresive miRNA in certain types of cancer.

In a study of the relationship between miR-221 and gastric cancer, Kim et al (22) reported that miR-221 downregulates both p27 and p57 that delay cell cycle. Chun-Zhi et al (23) reported that miR-221 upregulates gastric carcinoma cell proliferation and radioresistance by targeting the tumor suppressor gene PTEN. These findings support our results that miR-221 was overexpressed in the relapsed group.

There are no previous reports on miR-4732-5p and miR4758-3p expression. We found that both were highly expressed in gastric cancer patients who relapsed after adjuvant S-1 chemotherapy. We, therefore, consider both miR-4732-5p and $\mathrm{miR}-4758-3 \mathrm{p}$ expression to be related to recurrence after adjuvant S-1 chemotherapy. Further larger and more detailed studies are required to confirm these findings.

Combination of miRNAs is a very useful technique to improve the prediction of recurrence (24). From a group of 9 miRNAs (as shown in Table II), various permutations and combinations demonstrated that the combination of 4 miRNAs showed better positive and negative predictive values and predicted relapse more accurately after S-1 chemotherapy compared with a combination of other miRNAs. Although miR-221 was highly expressed in gastric cancer patients who relapsed after adjuvant S-1 chemotherapy, it was not useful in the prediction of relapse compared with the other 4 specific miRNAs.

In conclusion, this is the first report on miRNAs that are relapse-associated in gastric cancer patients after $\mathrm{S}-1$ adjuvant chemotherapy. In the present study, we identified 5 specific miRNAs (miR-92b, 422a, 4732-5p, 4758-3p and 221) related to gastric cancer recurrence after adjuvant $\mathrm{S}-1$ chemotherapy. The combination of 4 miRNAs, miR-92b, 422a, 4732-5p and 4758-3p, may possibly help to predict recurrence after S-1 chemotherapy.

\section{Acknowledgements}

We received research grants from the Japanese Ministry of Education, Culture, Sports, Science and Technology (MEXT/JSPS KAKENHI grant no. B: 23390320). We also received a research grant from the Japan Society for the Promotion of Science (JSPS) Funding Program for WorldLeading Innovative R\&D on Science and Technology (FIRST Program).

\section{References}

1. Terashima M, Kitada K, Ochiai A, Ichikawa W, Kurahashi I, Sakuramoto S, Katai H, Sano T, Imamura H and Sasako M: Impact of expression of human epidermal growth factor receptors EGFR and ERBB2 on survival in stage II/III gastric cancer. Clin Cancer Res 18: 5992-6000, 2012.

2. Fujimoto S, Akao T, Itoh B, Koshizuka I, Koyano K, Kitsukawa Y, Takahashi M, Minami T, Ishigami H, Miyazaki M, Amamiya K, Ohyama Y, Ono K, Kure M, Itoh Kenjiro and Hikosaka T: Protracted oral chemotherapy with fluorinated pyrimidines as an adjuvant to surgical treatment for stomach cancer. Ann Surg 185: 462-466, 1977.

3. Earle CC and Maroun JA: Adjuvant chemotherapy after curative resection for gastric cancer in non-Asian patients: revisiting a meta-analysis of randomized trials. Eur J Cancer 35: 1059-1064, 1999.

4. Mari E, Floriani I, Tinazzi A, Buda A, Belfiglio M, Valentini M, Cascinu S, Barni S, Labianca R and Torri V: Efficacy of adjuvant chemotherapy after curative resection for gastric cancer: a metaanalysis of published randomized trials. Ann Oncol 11: 837-843, 2000 .

5. Sakuramoto S, Sasako M, Yamaguchi T, Kinoshita T, Fujii M, Nashimoto A, Furukawa H, Nakajima T, Ohashi Y, Imamura H, Higashino M, Yamamura Y, Kurita A and Arai K: Adjuvant chemotherapy for gastric cancer with S-1, an oral fluoropyrimidine. N Engl J Med 357: 1810-1820, 2007.

6. Japanese Gastric Cancer Association: Japanese gastric cancer treatment guidelines 2010 (ver. 3). Gastric Cancer 14: 113-123, 2011.

7. Sasako M, Sakuramoto S, Katai H, Kinoshita T, Furukawa H, Yamaguchi T, Nashimoto A, Fujii M, Nakajima T and Ohashi Y: Five-year outcomes of a randomized phase III trial comparing adjuvant chemotherapy with S-1 versus surgery alone in stage II or III gastric cancer. J Clin Oncol 29: 4387-4393, 2011.

8. Calin $\mathrm{G}$ and Croce CM: MicroRNA signatures in human cancers. Nat Rev Cancer 6: 857-866, 2006.

9. Lu J, Getz G, Miska EA, Alvarez-Saavedra E, Lamb J, Peck D, Sweet-Cordero A, Ebert BL, Mak RH, Ferrando AA, Downing JR, Jacks T, Horvitz HR and Golub TR: MicroRNA expression profiles classify human cancers. Nature 435: 834-838, 2005.

10. Hummel R, Hussey DJ and Haier J: MicroRNAs: predictors and modifiers of chemo- and radiotherapy in different tumour types. Eur J Cancer 46: 298-311, 2010.

11. Koizumi W, Narahara H, Hara T, Takagane A, Akiya T, Takagi M, Miyashita K, Nishizaki T, Kobayashi O, Takiyama W, Toh Y, Nagaie T, Takagi S, Yamamura Y, Yanaoka K, Orita H and Takeuchi M: S-1 plus cisplatin versus S-1 alone for first-line treatment of advanced gastric cancer (SPIRITS trial): a phase III trial. Lancet Oncol 9: 215-221, 2008.

12. Osawa S, Shimada Y, Sekine S, Okumura T, Nagata T, Fukuoka J and Tsukada K: MicroRNA profiling of gastric cancer patients from formalin-fixed paraffin-embedded samples. Oncol Lett 2: 613-619, 2011.

13. Xing Y, Bronstein Y, Ross MI, Askew RL, Lee JE, Gershenwald JE, Royal R and Cormier JN: Contemporary diagnostic imaging modalities for the staging and surveillance of melanoma patients: a meta-analysis. J Natl Cancer Inst 103: 129-142, 2011.

14. Nakajima G, Hayashi K, Xi Y, Kudo K, Uchida K, Takasaki K, Yamamoto $\mathrm{M}$ and Ju J: Non-coding MicroRNAs hsa-let-7g and hsa-miR-181b are associated with chemoresponse to S-1 in colon cancer. Cancer Genomics Proteomics 3: 317-324, 2006.

15. Haug BH, Henriksen JR, Buechner J, Geerts D, Tømte E, Kogner P, Martinsson T, Flægstad T, Sveinbjørnsson B and Einvik C: MYCN-regulated miRNA-92 inhibits secretion of the tumor suppressor DICKKOPF-3 (DKK3) in neuroblastoma. Carcinogenesis 32: 1005-1012, 2011

16. Wang K, Wang X, Zou J, Zhang A, Wan Y, Pu P, Song Z, Qian C, Chen Y, Yang S and Wang Y: miR-92b controls glioma proliferation and invasion through regulating Wnt/beta-catenin signaling via Nemo-like kinase. Neuro Oncol 15: 578-588, 2013.

17. Ichikawa W, Takahashi T, Suto K, Shirota Y, Nihei Z, Shimizu M, Sasaki Y and Hirayama R: Simple combinations of 5-FU pathway genes predict the outcome of metastatic gastric cancer patients treated by S-1. Int J Cancer 119: 1927-1933, 2006.

18. Jeung HC, Rha SY, Shin SJ, Lim SJ, Roh JK, Noh SH and Chung HC: Predictive values of 5-fluorouracil pathway genes for S-1 treatment in patients with advanced gastric cancer. Anticancer Drugs 22: 801-810, 2011. 
19. Kai K, Kitajima Y, Hiraki M, Satoh S, Tanaka M, Nakafusa Y, Tokunaga O and Miyazaki K: Quantitative double-fluorescence immunohistochemistry (qDFIHC), a novel technology to assess protein expression: a pilot study analyzing 5-FU sensitive markers thymidylate synthase, dihydropyrimidine dehydrogenase and orotate phosphoribosyl transferases in gastric cancer tissue specimens. Cancer Lett 258: 45-54, 2007.

20. Gougelet A, Pissaloux D, Besse A, Perez J, Duc A, Dutour A, Blay JY and Alberti L: Micro-RNA profiles in osteosarcoma as a predictive tool for ifosfamide response. Int J Cancer 129: 680-690, 2011.

21. Faltejskova P, Svoboda M, Srutova K, Mlcochova J, Besse A, Nekvindova J, Radova L, Fabian P, Slaba K, Kiss I, Vyzula R and Slaby O: Identification and functional screening of microRNAs highly deregulated in colorectal cancer. J Cell Mol Med 16: 2655-2666, 2012.
22. Kim YK, Yu J, Han TS, Park SY, Namkoong B, Kim DH, Hur K, Yoo MW, Lee HJ, Yang HK and Kim VN: Functional links between clustered microRNAs: suppression of cell-cycle inhibitors by microRNA clusters in gastric cancer. Nucleic Acids Res 37: 1672-1681, 2009.

23. Zhang CZ, Han L, Zhang AL, Fu YC, Yue X, Wang GX, Jia ZF, Pu PY, Zhang QY and Kang CS: MicroRNA-221 and microRNA222 regulate gastric carcinoma cell proliferation and radioresistance by targeting PTEN. BMC Cancer 10: 367-376, 2010.

24. Zhang X, Yan Z, Zhang J, Gong L, Li W, Cui J and Liu Y: Combination of hsa-miR-375 and hsa-miR-142-5p as a predictor for recurrence risk in gastric cancer patients following surgical resection. Ann Oncol 22: 2257-2266, 2011. 